\title{
EI Desarrollo Profesional Docente en Hong Kong Comparado con los Países Anglosajones: El Rol del Confucianismo
}

\author{
LAM Bick Har \\ The Hong Kong Institute of Education (Hong Kong) \\ (Recibido el 20 de enero de 2015; Aceptado el 4 de abril de 2015)
}

\begin{abstract}
RESUMEN: El desarrollo profesional docente (DPD) ha sido el foco de numerosos estudios de investigación en los últimos años. Los investigadores buscan comprender el proceso del DPD, mientras que los profesionales se centran en diseñar políticas que faciliten el DPD. El consenso que emerge de la literatura es que el DPD debe ser 'individualizado': más centrado en fomentar el crecimiento personal de los profesores que en imponer 'prácticas estandarizadas'. Los países anglosajones han sido líderes en el campo del DPD, dando lugar a que muchos países imiten sus prácticas. Por medio de un análisis documental, este artículo revisa los principios del DPD en Hong Kong en los últimos cuarenta años y los compara con las prácticas típicamente adoptadas en los países anglosajones. El artículo sugiere que los principios del DPD en Hong Kong evolucionaron desde un modelo de 'entrenamiento del profesor', pasando por un modelo de 'énfasis en las habilidades genéricas' hasta llegar a la 'educación permanente'. El concepto de 'Cultura de Herencia Confuciana' es utilizado para explicar las diferencias entre los países anglosajones y Hong Kong en relación a sus prácticas de DPD. Se discuten implicaciones referidas a la adaptación de las prácticas de DPD interculturalmente.
\end{abstract}

Palabras clave: cultura, políticas educativas, Hong Kong, desarrollo profesional del docente

\section{Teacher Professional Development in Hong Kong Compared to Anglosphere: The Role of Confucian Philosophy}

\begin{abstract}
Teacher Professional Development" (aka teacher PD) has garnered research attention in recent years. Scholars seek to understand the teacher PD process, while practitioners are interested in deriving policies to facilitate teacher PD. The consensus emerging from the pool of literature is that teacher PD should be "individualized": more focused on fostering teachers' personal growth instead of enforcing 'standard practices'. The Anglosphere has been leading in the domain of teacher PD, causing many regions to imitate its practices. Using documentary analysis, this paper reviews teacher PD policies in Hong Kong over the past forty years and compares them with practices typically adopted in the Anglosphere. The paper suggests that the PD policies of Hong Kong progressed from
\end{abstract}


'solely teacher training', to 'emphasis on generic skills', to 'lifelong learning'. The concept of 'Confucian Heritage Culture' is used to explain the differences between the Angloshpere and Hong Kong in terms of PD practices. Implications for adapting teacher PD practices cross-culturally are discussed.

Keywords: culture, education policies, Hong Kong, teacher professional development

Correspondencia: LAM Bick Har, Associate Professor. Departamento de Curriculum e Instrucción. Instituto de Educación de Hong Kong. Room 32, Departamento de Curriculum e intrucción, Instituto de Educación de Hong Kong, 10 Lo Ping Road, Tai Po, Nuevos Territorios (Hong Kong). Email: bhlam@ied.edu.hk

\section{Cómo citar este artículo:}

Lam, B. H. (2015). El desarrollo profesional docente en Hong kong comparado con los países anglosajones: El rol del Confucianismo (trad. al castellano de J. M. Mateu Mateu). Psicología, Sociedad y Educación, 7(3), 405-422. [V.O.: Teacher professional development in Hong Kong compared to anglosphere: The role of Confucian philosophy. Psychology, Society and Education, 7(3), 295-310].

\section{Introducción}

El Desarrollo Profesional Docente (DPD) ha sido un fenómeno ampliamente estudiado en las Ciencias Sociales (p.ej., Guskey, 2002; Speck y Knipe, 2005). El término fue acuñado en principio por académicos del comportamiento organizacional para referirse a una adquisición individual de habilidades y conocimiento necesarios para avanzar en su carrera (Speck y Knipe, 2005), y es aplicado para estudiar los empleos de diversas ocupaciones, incluidos los supervisores de enfermería (Neary, 2000), científicos (Hunter, Laursen y Seymour, 2007) y profesores (Guskey, 2002). Concretamente, los estudios del DPD han llamado la atención académica de los educadores. En Educación, se ha formado un campo 'El Desarollo Profesional del Docente' (también conocido como 'El Desarrollo del Docente' o 'DP del Docente').

Actualmente, el DPD es definido como un proceso a través del cual un profesor adquiere las habilidades y el conocimiento que necesita para avanzar en su carrera (Guskey, 2002), y cuenta con un conjunto de teorías propias. Sabemos que el DPD está relacionado con diversos resultados del trabajo del profesor: los estudios han encontrado que el progreso en la carrera profesional se relaciona positivamente con el compromiso en el trabajo (Day y Gu, 2007), y que los docentes más avanzados tienden a tener estudiantes que más exitosos académicamente (Caprara, Barbaranelli, Steca y Malone, 2006). Mientras tanto, el sistema de educación de Hong Kong es víctima de dos problemas: los profesores de escuelas locales informan padecer gran cantidad de estrés que perjudica su compromiso con el trabajo (Choi y Tang, 2009); además, aunque Hong Kong ha tenido resultados superiores a la media en las pruebas internacionales, el descenso de nivel de sus estudiantes en el dominio de idiomas y otras disciplinas ha sido una queja constante de los educadores locales (Chan, 2002). 
Como consecuencia, la investigación en DPD ha atraído la atención de los educadores en Hong Kong (Chan, 1998; Lam, 2014).

Este artículo permitirá (1) revisar las tendencias del DPD en los países anglosajones (el mundo de habla inglesa incluye los Estados Unidos, Australia, Canadá, Nueva Zelanda y Reino Unido); (2) compararlos con las prácticas de Hong Kong; y (3) suscitar ideas de las cuales otras regiones podrían aprender. Comparando las prácticas de DPD en Hong Kong y en el mundo anglosajón, destacaremos las características clave del DPD en Hong Kong. También puntualizaremos las claves específicas a las 'Culturas de Herencia Confuciana' (CHC's). A través de todo ello, identificaremos los problemas que surgen de adaptar las prácticas de los países anglosajones a otras culturas y discutiremos las posibles soluciones.

\section{La Nueva Tendencia del DPD en el Mundo Anglosajón}

Un gran número de estudios se han realizado sobre el DPD, centrados en diversos aspectos (Avalos, 2011). Todos ellos, sin embargo, han experimentado un cambio de paradigma, ya que el DPD es visto cada vez más como un proceso que implica crecimiento y desarrollo a largo plazo, y no únicamente como programas de formación previos al servicio y durante el servicio docente (Walling y Lewis, 2000). Dentro de este modelo, los profesores son vistos como aprendices activos y profesionales reflexivos que están motivados a colaborar para aprender (DarlingHammond y Richardson, 2009), y se entiende que aprendizaje de los profesores es contextualizado, en vez de centrarse a un conjunto de 'medidas' estándar aplicadas en todos los casos (McLaughlin y Zarrow, 2001). Para ser más precisos, se trata de una aproximación al DPD que atiende más a la persona.

Esta tendencia ha dado lugar a cambios en la investigación y en las prácticas del DPD. En el pasado, la investigación y las prácticas a menudo enfatizaban el 'DPD como adquisición de conocimientos' (Shulman, 1986; 1987). Thiessen (2000), por ejemplo, explicó que "En el corazón de esta orientación está la imagen de la enseñanza como un trabajo sobre el conocimiento. Tal trabajo [...] implica el uso interrelacionado del conocimiento práctico (rutinas, procedimientos, procesos) y el conocimiento proposicional (disciplina basada en las teorías y en los conceptos, principios pedagógicos, proposiciones específicas a una situación)" (p. 528). Como consecuencia, en las prácticas tempranas del DPD (antes de la década de los 80) abundaban los talleres y los programas de formación (Ben-Peretz, 2011). Además, se produjeron discusiones sobre la extensión, duración e intensidad de la formación inicial del profesor en los países de habla inglesa (p.ej., Cobb, 1999).

En contraste, el DPD posterior (de la década de los 90 en adelante) comenzó a enfatizar cómo los profesores podían mejorar continuamente sus habilidades de enseñanza con base en la experiencia. Las prácticas estándar incluyen la investigación-acción (Ross y Bruce, 2007), donde los profesores son invitados a realizar estudios de pequeña escala en los que evalúan la efectividad de sus prácticas de enseñanza, en vez de aplicar prácticas estándar aprendidas en cursos de formación del profesorado. Este nuevo modelo también tiene un elemento de 'colaboración' 
dado que incluye prácticas de tutoría (Hagger, McIntyre y Wilkin, 2013), un proceso de relación basado en la comunicación donde un 'mentor' transmite conocimiento y da apoyo psicológico a un 'aprendiz'.

La investigación que evalúa los resultados del DPD sugiere que las nuevas prácticas, incluso la auto-reflexión (McKernan y McKernan, 2013), la investigaciónacción (Stringer, 2007) y la tutoría (Hagger et al., 2013) han sido útiles a la hora de fomentar entre los profesores un mayor nivel de compromiso en el trabajo y un mayor rendimiento. Como ejemplos, Bustingorry (2008) evalúo a un grupo de profesores chilenos que recibieron formación en investigación-acción, encontrando mejores calificaciones en los estudiantes enseñados por dichos profesores. La revisión de Hagger y colaboradores (2013) sobre prácticas de tutorías también indicaron que éstas son generalmente útiles para la mejora del desempeño docente. En líneas generales, este conjunto de nuevas prácticas de DPD ha sido exitoso. Como resultado, actualmente muchas regiones (incluyendo Hong Kong) están adaptándose sus prácticas de DPD a las nuevas tendencias.

\section{EI DPD en Hong Kong}

La principal fuente de datos del presente artículo son los documentos de política educativa publicados por el gobierno de Hong Kong en los últimos 40 años. Los textos pertinentes se hallaron en los sitios webs oficiales del (1) Departamento de Educación, (2) de la Comisión de Educación, y (3) la Comisión sobre Desarrollo Profesional de los Directores y Docentes (COTAP, anteriormente el Comité Consultivo sobre la Educación y Capacitación del Docente, ACTEQ). También tuvimos en cuenta los resultados de las encuestas que el gobierno de Hong Kong en 2006 y 2009 realizó para evaluar la efectividad del DPD. Aunque los resultados de las dos encuestas no pudieron ser comparados sistemáticamente entre sí, dado que sus formatos eran diferentes, estos resultados ofrecen una visión sobre el contexto del DPD en Hong Kong. Todos estos documentos y las declaraciones de propósitos de los tres órganos de gobierno arriba citados fueron considerados como datos de la presente revisión.

\section{La trayectoria del DPD en Hong Kong}

Nuestra revisión del DPD de Hong Kong en las últimas cuatro décadas mostró que este país ha seguido una trayectoria similar a la del mundo anglosajón. Inicialmente (los años 80 y anteriormente), el gobierno de Hong Kong trató el DPD como 'entrenamiento del profesorado'. Los cursos de formación de las instituciones locales eran las únicas prácticas de DPD. Los profesores eran vistos como trabajadores que requerían ser formados, como se indica explícitamente en el Libro Blanco producido por el gobierno de Hong Kong en 1978: "otros objetivos deben también tenerse en cuenta en el informe, concretamente asegurar una provisión regular de profesores cualificados para ocuparse de los puestos docentes mientras presentan y proveen de un entrenamiento básico a los profesores en activo sin experiencia" (p. 13; nuestra traducción). A mediados de la década de los 80, el 
sistema de educación de Hong Kong (incluyendo la división especializada en DPD) fue criticado por el comité visitante (1982), que llevó a cabo una serie de observaciones en distintos colegios de Hong Kong. Un punto importante planteado por el comité fue que la educación de Hong Kong se centraba casi totalmente en el profesor y colocaba un énfasis excesivo en la 'cantidad' (formar más profesores y producir más estudiantes) en detrimento de la 'calidad' (producir una educación de calidad alta).

En respuesta, en la década de los 90 el gobierno de Hong Kong comenzó a implantar nuevas políticas para incrementar la calidad de su sistema educativo. Por primera vez, distintas intervenciones educativas que no se centraban en las calificaciones de los estudiantes, sino en su desarrollo no académico, se convirtieron en parte de la política oficial (Educational and Manpower Branch, 1993). Para atender a esta situación, el DPD comenzó a orientarse a dotar a los profesores de habilidades generales (Education Commission, 2000). Se comenzó a requerir que los profesores desarrollaran habilidades relacionadas con la orientación, las tecnologías de la información y con los idiomas, terminando con exámenes en los que eran evaluados sobre estas competencias. Los centros de recursos fueron creados para ofrecer a los profesores materiales de enseñanza ejemplares. Estos abrieron el camino del cambio hacia un enfoque del DPD más avanzado; como se manifiesta en la Comisión de Educación (2000), en la década del 2000 las nuevas medidas atenderían a "la promoción del desarrollo profesional de los docentes y a mejorar su profesionalismo, su sentido del compromiso y su entusiasmo" (p. 151). Así, en la década del 2000, el nuevo paradigma del DPD tomó gran impulso.

\section{El DPD de Hong Hong tras el año 2000}

El Departamento de Educación de Hong Kong propuso el Programa de Inducción Docente a partir del 2005 en adelante (Education Bureau, 2014a). Dicho programa está dirigido al primer año de los profesores recién titulados. Se espero que las escuelas y los profesores más sénior le provean "de estímulo y apoyo, de manera que también disfruten de las experiencias de aprendizaje positivas y de un comienzo agradable para sus carreras", a través de actividades con "un elemento de autoreflexión", de instrumentos que sirvan para "registrar cada trayectoria del desarrollo de los profesores principiantes", de evaluaciones que "informen del futuro desarrollo de los programas de inducción de los colegios", y de los programas de tutoría (p. 2, Education Bureau, 2014a; nuestra traducción). Este plan no dictó oficialmente un conjunto de procedimientos, sino más bien aconsejó el uso de las mismas técnicas sugeridas por la ACTEQ (2009a). Las directrices de la ACTEQ incluían acciones específicas que las escuelas podrían realizar en la inducción de sus profesores principiantes y que han demostrado empíricamente ser útiles.

Otro de los temas clave en las prácticas del DPD en Hong Kong es el establecimiento de redes sociales entre profesores. La planificación de la enseñanza colaborativa (Education Bureau, 2012), por ejemplo, da pie a los profesores a participar en reuniones programadas en las que planean sus lecciones 
colaborativamente, realizando reflexiones sobre la enseñanza, con el objetivo de promover el intercambio de ideas entre profesores, promoviendo de este modo el DPD. El Departamento de Educación también ha añadido también sesiones de intercambio tanto dentro como fuera de la escuela (2010). Cada año, los profesores de multitud de colegios ofrecerán sesiones de intercambio sobre temas específicos para informar a otros profesores sobre sus propias prácticas. Para fortalecer aún más dichas redes, el Departamento de Educación también propuso programas donde los profesores de las escuelas de Hong Kong eran enviados a enseñar en colegios de China continental y Taiwán para aprender sobre los sistemas de educación y las técnicas de estas regiones (p.ej., Education Bureau, 2014b).

Las prácticas relacionadas con los dos temas anteriores han sido recibidas de forma favorable. De acuerdo con la encuesta de ACTEQ de 2006, sobre el 80\% de los profesores de Hong Kong respondieron que las prácticas citadas habían sido implantadas en sus colegios, y más del $80 \%$ de ellos contestaron que las consideraban útiles. Más del $90 \%$ de los encuestados respondieron que las prácticas les habían ayudado a: (1) mejorar sus propias competencias docentes; (2) aumentar la eficacia del aprendizaje y la enseñanza; (3) aumentar su comprensión del conocimiento de la materia; y (4) mejorar su capacidad para tratar con las diversas necesidades de aprendizaje de los estudiantes. Parece por tanto que estas prácticas fueron un éxito global.

Se ofreció a los profesores de Hong Kong formación relacionada con estos dos temas. Por ejemplo, se ofrecieron talleres que formaban a profesores en tutoría, como parte del Plan de Inducción Docente (Education Bureau, 2014a). Desde principios del 2000, también se han ofrecido cursos especializados sobre liderazgo (Hong Kong Institute of Education 2014). La tradición de trabajar para mejorar las aptitudes de los profesores está en progreso. Por ejemplo, del 2000 en adelante, los profesores deben obtener certificados reconocidos para poder ser contratados por las escuelas (Education Bureau, 2014c). En los noventa, muchos profesores no estaban cualificados pero se les permitía enseñar debido a la escasez de profesionales. Por el contrario, hoy día más del $95 \%$ de los profesores de primaria y secundaria en los colegios de Hong Kong cumplen todos los requisitos exigidos (Education Bureau, 2014d).

\section{Diferencia de las Prácticas de Hong Kong con las Anglosajonas}

El Anexo 1 resume las principales prácticas de DPD ofrecidas por el Departamento de Educación. A grandes rasgos, las prácticas del DPD en Hong Kong se parecen pero también difieren en muchos aspectos de las de los países anglosajones. Al igual que en los países anglosajones, el DPD en Hong Kong comienza como 'mero entrenamiento', pero una vez que surgió la crítica contra dicho enfoque, el gobierno respondió mediante con la implementación de prácticas del DPD que enfatizaban el crecimiento y el desarrollo de los profesores a largo plazo. Para lograr este objetivo, Hong Kong trabajó en la creación de lazos cooperativos entre las escuelas y las universidades (Wise, 2000), que servían como proveedores de programas y talleres 
de desarrollo profesional. El contenido de esta formación es similar al anglosajón. También Hong Kong usa ampliamente el aprendizaje colaborativo junto con la tutoría, dos enfoques comúnmente adoptados en países anglosajones (Sandholtz, 2000).

En general, las prácticas del DPD en Hong Kong se parecen a las que describe la literatura dominante, aunque con una diferencia crítica: la falta de auto-dirección en Hong Kong. Pese a la mayoría de las prácticas comunes en la literatura han sido adoptadas por Hong Kong en cierto grado, como explicamos anteriormente, las prácticas que incluyen un componente de 'autonomía' en el desarrollo profesional están notablemente ausentes. Glatthorn (1987), por ejemplo, describió la idea de 'desarrollo colegial', donde los profesores debaten sobre cuestiones profesionales con interés personal con sus colegas (p.ej., cómo ellos mismos, en tanto individuos, deberían desarrollarse), con el objetivo de diseñar sus propios planes de desarrollo profesional individual. Clarke (1995) más tarde propuso el modelo del profesional reflexivo, en el que se espera que los profesores sean curiosos en la práctica. Surgiendo de estas ideas, un tema recurrente de los métodos posteriores del DPD es que a los profesores se les debe dar autonomía en su desarrollo. A este respecto, sin embargo, Hong Kong es bastante deficiente. Jin, Yeung, Tang y Low (2008) investigaron las fuentes del estrés para los profesores de institutos de Hong Kong. La fuente común más citada era las altas expectativas de los colegios y de los padres. Otros estudios de entrevista sugirieron que la densa reforma del currículum condujo a los colegios a 'competir' respecto a la formación de sus profesores, ejerciendo una cantidad notable de estrés sobre ellos (p.ej., Chan, 1998; Tang, Au, Schwarzer y Schmitz, 2001).

En esta misma línea, un estudio reciente de Hargreaves y colaboradores (2013) ha encontrado que aunque los docentes de Hong Kong generalmente consideran el aprendizaje colaborativo de forma positiva, ellos alegaron niveles menores de autonomía percibida (p.ej., menos inclinación a pensar en su participación como voluntario) en comparación a profesores de Londres. Los resultados sugieren una tendencia general de diferentes agentes educativos a presionar a los profesores para participar en ciertas actividades de DPD. Como consecuencia, la situación es que muchos profesores en Hong Kong (si no todos) no tienen mucha autonomía sobre su propio DPD, lo que puede degenerar en una competencia entre centros por formar a sus profesores.

Mientras los docentes de Hong Kong en su mayoría están involucrados y comprometidos con sus carreras profesionales (Choi y Tang, 2009), existe una preocupación subyacente respecto al impacto a largo plazo del DPD desarrollado por el Departamento de Educación. Esto es especialmente cierto considerando que los efectos negativos del estrés laboral sobre el compromiso con el trabajo son frecuentemente percibidos sólo conforme pasa el tiempo (Lam, 2011, 2012), y que las nuevas prácticas de DPD en Hong Kong han sido desarrolladas muy recientemente (Kennedy, 2005). Aunque las nuevas medidas implantadas por el 
gobierno de Hong Kong fueron razonablemente bien recibidas, tienen sus propios problemas subyacentes y limitaciones.

\section{Cómo la Cultura Moldea el DPD en Hong Kong}

Hong Kong es un 'Cultura de Herencia Confuciana' (CHC), cuyos valores están influenciados por la filosofía de Confucio (Penfold y van de Veen, 2014). Como hemos visto en nuestro resumen, la actual tendencia de las prácticas de DPD en Hong Kong son coherentes con el patrón esperable de una CHC que intenta adoptar las prácticas occidentales. Como propuso explícitamente la Comisión de Educación (2000): "nuestro sistema de educación está impregnado de la esencia de las culturas orientales y occidentales, preservando los elementos básicos de la educación china tradicional mientras adquiere los conceptos más avanzados, teorías y experiencias de una educación occidental moderna" (p. 2); "el objetivo a largo plazo de Hong Kong no es sólo convertirse en una de las ciudades más destacadas en China, sino también convertirse en una ciudad internacional democrática y civilizada que acoge la esencia cultural del Este y del Oeste." (p. 28; nuestra traducción). Esto indica el esfuerzo consciente por amoldar las prácticas del DPD occidentales a la cultura y los valores orientales. En las CHC's, los sistemas de educación están moldeados por la filosofía de Confucio, cuya influencia sobre el comportamiento de los profesores y los estudiantes es ampliamente conocida y estudiada (p.ej., Nguyen, Terlouw y Pilot, 2006). Analizando cómo la CHC ha afectado las prácticas de DPD en Hong Kong, el presente estudio destaca que Hong Kong ha puesto énfasis en los talleres y cursos de formación, así como en las redes sociales de los profesores. Sin embargo, esto también podría haber producido los aspectos más problemáticos de las prácticas de Hong Kong.

Primero, la CHC habría explicado por qué Hong Kong aceptó fácilmente las necesidades de talleres y cursos de formación en el DPD. El núcleo de dicha cultura es el Confucianismo. El trabajo de los filósofos Confucianos titulado 'Las Tres Características Clásicas' explica explícitamente su mensaje: "Se dan recompensas por trabajar, pero no se dan recompensas por jugar". Tradicionalmente, el arquetipo de hombre confuciano está bien formado en filosofía y humanidades, muy diferente al arquetipo de hombre occidental que es más semejante a un caballero; el prototipo de hombre occidental muestra caballerosidad, coraje, fuerza física e ingenio ( $\mathrm{Li}$, 2003). Moldeada por estos ideales, las CHC's tales como Hong Kong, China, Corea y Japón son bien conocidas por su gran obsesión por las actividades académicas, llevadas a cabo frecuentemente mediante aprendizaje memorístico (Biggs, 1990, 1998). No es sorprendente que Hong Kong fácilmente pudiera entender la necesidad de proponer talleres y cursos de formación del profesor para promover su DPD, como se muestra en la revisión. De hecho, en las estadísticas de la ACTEQ (2009b), las respuestas de los profesores sugieren que éstos preferían dicho aprendizaje. Más del $90 \%$ de los profesores dijeron encontrar las actividades de 'estructuradas' de aprendizaje (tales como los talleres y cursos) útiles para su DPD, convirtiendo estas actividades en las más populares de entre ofrecidas por el gobierno de Hong Kong. 
Otra característica de las CHC's es el énfasis general por las relaciones sociales. El Confucianismo da tremenda importancia a las 'Cinco Relaciones Básicas de la Sociedad' - un gobernador y sus súbditos, padres e hijos, hermanos mayores y menores, maridos y esposas, y amigos. Confucio y sus seguidores destinaron grandes esfuerzos para promover los comportamientos adecuados en estas relaciones. Por ejemplo, el 'Libro de la Piedad Filial', una larga serie de códigos que Confucio produjo para sugerir cómo los niños deberían relacionarse con sus padres, propone que mientras los padres están obligados a proteger y proporcionar orientación moral a sus hijos pequeños, a los hijos adultos les corresponde apoyar a sus padres una vez que éstos sean ancianos. Trabajos similares han sido producidos en relación a las otras cuatro relaciones básicas. Es por ello que a menudo se dice que el fundamento de las CHC radica en las relaciones entre los individuos (p.ej., Ip, 1996).

El budismo, otra filosofía dominante entre las CHC's, también enfatiza la importancia de las relaciones, aunque de un modo más metafísico. El budismo enseña que a través de prácticas de piedad (p.ej., estudios de las escrituras y la meditación), los seres humanos pueden acercarse a la Iluminación, que es necesaria para alcanzar el Nirvana, un estado de liberación final del sufrimiento y, por tanto, el estado más elevado que un ser puede alcanzar de acuerdo con las creencias Budistas. Aunque una etapa crucial antes de la Iluminación es convertirse en un Bodhisattva, un estatus donde el ser se esfuerza en ayudar a otros seres en sus caminos para alcanzar la Iluminación. Incluso el propio Buda, en la tradición budista, contaba que solía ser un Bodhisattva antes de lograr la verdadera Iluminación. La veneración de los Bodhisattva es una práctica común de los Budistas que pertenecen a las CHC's (Kariyawasam, 2002). Por consiguiente, no es sorprendente que las personas de las CHC's se declaren más preocupadas por las relaciones que aquellas que no pertenecen a CHC's. Los miembros de CHC's son bastante más propensos a atender a las relaciones (Gold, Guthrie y Wank, 2002), y a verse a sí mismos como miembros de grupos en vez de como individuos (Triandis, 2001). Esto explica en parte por qué Hong Kong, siendo una CHC, ha utilizado tantas prácticas de DPD relacionadas con las redes sociales. El 'Plan de Inducción Docente', los 'Planes de Lecciones de Colaboración', y los 'Programas de Tutorías' incluyen elementos de las redes sociales. Los profesores locales también han respondido favorablemente a estas prácticas, con más del 70\% indicando que la tutoría es útil para su desarrollo (LópezReal y Kwan, 2005). La encuesta de la ACTEQ (2006) sugirió que el 88\% de los profesores en Hong Kong se habían implicado en observaciones de clases de sus compañeros y el $84 \%$ habían participado en colaboraciones con colegas, como preparando clases conjuntamente, reflejando alta disposición a participar en prácticas orientadas a fomentar relaciones entre los docentes.

Sin embargo, estos elementos de las CHC's probablemente han contribuido también a los aspectos más problemáticos de las prácticas del DPD en Hong Kong. Como se esperaría de las culturas que enfatizan las relaciones, las CHC's también tienden a minimizar o directamente suprimir las expresiones de individualidad (Triandis, 2001). Como se explica en Lam (2011): 
La nueva demanda de los profesores de Hong Kong para involucrarse en procesos de investigación -un enfoque constructivista y centrado en el estudiante- les ha planteado un gran desafio dado que está en conflicto con el rol tradicional de los profesores y estudiantes en las clases de China, donde el docente es la autoridad y los estudiantes reciben conocimiento de los libros de forma pasiva. (p. 269; nuestra traducción).

Esto puede explicar por qué Hong Kong ha ignorado la importancia de proveer a los docentes con más autonomía. Mientras la última tendencia de las prácticas del DPD anima a los profesores a diseñar sus propios planes de desarrollo profesional, las prácticas del DPD en Hong Kong tiene pocos (o ninguno) de estos elementos. Al contrario, las medidas que el Departamento de Educación ha implantado están mayormente preocupadas con 'elevar el nivel' (p.ej., requiriendo a los profesores tener ciertas calificaciones o alcanzar ciertos criterios de rendimiento arbitrario). También las expectativas de los diferentes agentes educativos son una fuente mayor de estrés para los profesores en Hong Kong (Jin et al., 2008). Esto es probablemente un razón fundamental que explica por qué Hong Kong adoptó nuevas aproximaciones al DPD de forma tan tardía, en comparación con otros países del mundo. Pese a no estar especialmente anticuado, Hong Kong no ha favorecido prácticas tales como la tutoría y el aprendizaje colaborativo hasta bien entrada la década del 2000. Para entonces, tales prácticas era ya normativas en los países anglosajones (p.ej., VillegasReimers, 2003). La obsesión de las CHC's por 'mejorar los resultados' pueden haber hecho difícil aceptar la existencia de necesidades de estudiantes y docentes respecto a su desarrollo no académico. Así, sugerimos que muchas de las propiedades del DPD en Hong Kong pueden atribuirse a su estatus como CHC.

\section{Lecciones Aprendidas de las Prácticas de DPD de Hong Kong}

En conjunto, Hong Kong ha intentado adaptar las prácticas de DPD de los países anglosajones para adaptarse a las nuevas tendencias. Sin embargo, no ha adoptado cualquier práctica sino que ha sido selectivo en las medidas adoptadas. Concretamente, Hong Kong ha hecho grandes esfuerzos por dar entrenamiento a los profesores en ejercicio, en la inducción del profesorado novel y en crear amplias redes escolares. Los profesores locales respondieron favorablemente a estas medidas, especialmente a la inducción del docente y a las redes de escolares (López-Real y Kwan, 2005; Kwan y López-Real, 2005). En la teoría de la auto-determinación (Ryan y Deci, 2000), las relaciones podrían motivar a los humanos a hacer una tarea simplemente por el propio placer asociado con ésta. Cuando una figura cálida de apoyo está presente durante una determinada tarea, los seres humanos experimentan una sensación de seguridad porque la presencia de dicha figura indica disponibilidad de apoyo (Deci y Ryan, 2008).

A su vez, comenzarían a estar más dispuestos a asumir riesgos y aprender a través del ensayo-error. Esta explicación ha sido empíricamente apoyada por el aumento del efecto motivacional asociado a la presencia de otros (Niemiec y Ryan, 
2009). Aunque este efecto ha sido ampliamente demostrado en los países anglosajones, podría ser particularmente importante para los miembros de las CHC's. Esto se debe a que los miembros de las CHC's demuestran están más necesitados del apoyo y de la confianza de los demás, así como de mostrarse conformes con las opiniones del grupo (Triandis, 2001). Son también más vulnerables a los efectos negativos de la ausencia de apoyos. Considerando esto, prácticas como la inducción docente y la tutoría serían especialmente adecuadas para Hong Kong, y de forma más general, para las distintas CHC's. Esto sugiere que cuando otras CHC's (como China, Taiwán y Singapur) adaptan prácticas del DPD provenientes de países anglosajones, deberían atender a las prácticas basadas en las redes sociales y en las relaciones entre los profesores. En mayor escala, sugerimos que las diferentes partes del mundo no deben usar a ciegas estas prácticas mostradas útiles para otros países, sino que deben considerar lo que motiva a las personas de sus culturas. Por ejemplo, los profesores de EE.UU. y de Australia pueden ser más abiertos al desarrollo auto-dirigido en comparación a sus homólogos en las CHC's, porque los miembros de estas culturas son bastante más propensos a necesitar autonomía y auto-afirmación que a seguir las normas en la toma de decisiones (Rhee, Uleman, Lee y Roman, 1995).

Además, un fenómeno observado en Hong Kong es que las prácticas del DPD han producido estrés y ansiedad en los profesores principalmente debido a las altas expectativas de los agentes educativos (Jin et al., 2008). Esto puede explicarse por la tendencia de las CHC's a dar gran importancia a 'dar buena cara' y al miedo a parecer incompetente (Thoma, McNaught, Wong y Li, 2011). Por el contrario, las causas más comunes de estrés para los profesores de EE.UU. y Australia son los motivos salariales y las dificultades de promoción (p.ej., Zhai, Raver y Li-Grining, 2011). Esto implica que cuando se trata del problema del estrés docente, las CHC's deben considerar distintas aproximaciones de las utilizadas por los países anglosajones. Un solución posible sería el entrenamiento en autoestima para los profesores. Uno de los modos en que los miembros de culturas colectivistas como las CHC's divergen de sus homólogos más individualistas (p.ej., los países anglosajones) es que los primeros tienden a tener una autoestima más baja (Twenge y Crocker, 2002). La autoestima es esencial para asegurar la competencia individual, y por tanto para amortiguar la ansiedad - un estado en que la persona experimenta emociones negativas producidas por la incertidumbre sobre si podrá conseguir lo que quiere. También, una persona con alta autoestima tiende a estar confiada sobre su competencia, por lo tanto, más resistente contra los juicios negativos (Kumpfer, 2002). Así, el entrenamiento en autoestima puede ser posiblemente de ayuda a los profesores de Hong Kong, y una vez más, probablemente para los profesores de las CHC's en general. En este punto, es importante recordar que los países anglosajones están ya tomando acciones para abordar el problema del alto nivel de estrés laboral declarado entre los profesores (p.ej., Collie, Shapka y Perry, 2012). Sugerimos a este respecto que no es muy apropiado para las CHC's adaptar directamente las prácticas de los países anglosajones. En su lugar, deben diseñar sus propias prácticas para atender a las necesidades propias de su cultura. 


\section{Conclusiones Finales}

Actualmente, existe una tendencia al cambio en las prácticas de DPD de los países anglosajones, que han comenzado a ser más individualizadas y menos focalizadas en las 'prácticas estándar'. En respuesta a esta tendencia, el sistema educativo de Hong Kong adoptó algunas de las nuevas prácticas. Específicamente, se adoptaron la inducción docente, el establecimiento de redes sociales entre profesores, y la formación de los profesores locales en las habilidades requeridas. Las autoridades educativas de Hong Kong, aunque no adaptan ciegamente las prácticas de los países anglosajones, sí son explícitas en sus intentos de preservar los valores y la cultura orientales. Con esto en mente, hemos identificado cómo tales valores y culturas han influido en las prácticas del DPD en Hong Kong, así como las respuestas de los profesores a dichas iniciativas. Identificamos la falta de auto-dirección como rasgo característico del DPD en Hong Kong, y discutimos qué podrían aprender de nuestro análisis los sistemas educativos de otros países en el mundo.

\section{Referencias}

Advisory Committee on teacher Education and Qualifications. (2006). Task Force on Teachers' Continuing Professional Development. Hong Kong: Advisory Committee on teacher Education and Qualifications.

Advisory Committee on teacher Education and Qualifications. (2009a). Professional Development for Beginning teachers - An Induction Tool Kit (5th ed.). Hong Kong: Advisory Committee on teacher Education and Qualifications.

Advisory Committee on teacher Education and Qualifications. (2009b). Towards a Learning Profession: The teacher Competencies framework and the Continuing Professional Development of teachers. Hong Kong: Advisory Committee on teacher Education and Qualifications.

Avalos, B. (2011). Teacher professional development in Teaching and teacher Education over ten years. Teaching and teacher Education, 27(1), 10-20.

Ben-Peretz, M. (2011). Teacher knowledge: What is it? How do we uncover it? What are its implications for schooling?. Teaching and teacher Education, 27(1), 3-9.

Biggs, J. (1998). The assessment scene in Hong Kong. In P. Stimpson \& P. Morris (Eds.), Curriculum and Assessment for Hong Kong: One system, two components. (pp. 315-324). Hong Kong: Open University of Hong Kong.

Biggs, J. B. (1990). Asian students' approaches to learning: implications for teaching overseas students. In Australasian Tertiary Learning Skills and Language Conference. Brisbane.

Bustingorry, S. O. (2008). Towards teachers' professional autonomy through action research. Educational Action Research, 16(3), 407-420.

Caprara, G. V., Barbaranelli, C., Steca, P., \& Malone, P. S. (2006). Teachers' self-efficacy beliefs as determinants of job satisfaction and students' academic achievement: A study at the school level. Journal of School Psychology, 44(6), 473-490.

Chan, D. W. (1998). Stress, coping strategies, and psychological distress among secondary school teachers in Hong Kong. American Educational Research Journal, 35(1), 145- 163. 
Chan, E. (2002). Beyond pedagogy: Language and identity in post-colonial Hong Kong. British Journal of Sociology of Education, 23(2), 271-285.

Chan, S. (1999). The Chinese learner - a question of style. Education and Training, 41(6/7), 294304.

Choi, P. L., \& Tang, S. Y. F. (2009). Teacher commitment trends: Cases of Hong Kong teachers from 1997 to 2007. Teaching and teacher Education, 25(5), 767-777.

Clarke, A. (1995). Professional development in practicum settings: reflective practice under scrutiny. Teacher and teacher Education, 11(3), 243-261.

Cobb, V. (1999). An International Comparison of teacher Education. ERIC Digest. Wash- ington, DC: ERIC Clearinghouse on Teaching and teacher Education.

Collie, R. J., Shapka, J. D., \& Perry, N. E. (2012). School climate and social-emotional learning: Predicting teacher stress, job satisfaction, and teaching efficacy. Journal of Educational Psychology, 104(4), 1189-1204.

Darling-Hammond, L., \& Richardson, N. (2009). Research Review/Teacher Learning: What Matters. Educational Leadership, 66(5), 46-53.

Day, C., \& Gu, Q. (2007). Variations in the conditions for teachers' professional learning and development: sustaining commitment and effectiveness over a career. Oxford Review of Education, 33(4), 423-443.

Deci, E. L., \& Ryan, R. M. (2008). Self-determination theory: A macrotheory of human motivation, development, and health. Canadian Psychology/Psychologie canadienne, 49(3), 182-185.

Education Bureau. (2010). Professional development - Primary teachers sharing seminar. (Chinese), Retrieved on Dec 24, 2014, from

Education Bureau. (2012). School-based Curriculum Development in the Primary Schools Collaborative Lesson Planning. Retrieved on Dec 24, 2014, from http://www.edb.gov.hk/en/edu-system/primary-secondary/applicable-to-primarysecondary/sbss/school-based-curriculum-primary/our-work/collaborative-lessonplanning/p1.html

Education Bureau. (2014a). Pamphlet on ACTEQ's teacher Induction Scheme. Retrieved on Dec 24, 2014, from:

Education Bureau. (2014b). School Support Partners (Seconded teacher) Scheme SSP. Retrieved on Dec 24, 2014, from

Education Bureau. (2014c). Teacher Training and Qualifications. Retrieved on Dec 24, 2014, from

Education Bureau. (2014d). Figures and Statistics: Primary Education. Retrieved on Dec 24, 2014, from http://www.edb.gov.hk/en/about-edb/publications-stat/figures/pri.html

Education Commission. (2000). Reform Proposal for the Education System in Hong Kong. Retrieved on Jan 6, 2014, from

Educational and Manpower Branch. (1993). School Education in Hong Kong: A Statement of Aims. Retrieved on Jan 6, 2014, from

Glatthorn, A. A. (1987). Cooperative Professional Development: Peer-Centered Options for teacher Growth. Educational Leadership, 45(3), 31-35.

Gold, T., Guthrie, D., \& Wank, D. (Eds.). (2002). Social connections in China: Institutions, culture, and the changing nature of guanxi (No. 21). Cambridge: Cambridge Univer- sity Press.

Guskey, T. R. (2002). Professional development and teacher change. Teachers and Teaching: 
Theory and Practice, 8(3), 381-391.

Hagger, H., McIntyre, D., \& Wilkin, M. (Eds.). (2013). Mentoring: perspectives on school-based teacher education. London: Routledge.

Hargreaves, E., Berry, R., Lai, Y. C., Leung, P., Scott, D., \& Stobart, G. (2013). Teachers' experiences of autonomy in Continuing Professional Development: teacher Learning Communities in London and Hong Kong. Teacher Development, 17(1), 19-34.

Hong Kong Government. (1978). The Development of Senior Secondary and Tertiary Education. Retrieved on Jan 14, 2014, from

Hong Kong Institute of Education. (2014). Programme information. Retrieved on Nov 20, 2014, from http://www.ied.edu.hk/acadprog/pdp/programme.htm

Hunter, A. B., Laursen, S. L., \& Seymour, E. (2007). Becoming a scientist: The role of undergraduate research in students' cognitive, personal, and professional development. Science Education, 91(1), 36-74.

Ip, P. K. (1996). Confucian Familial Collectivism and the Underdevelopment of the Civic Person. In N. K. Lo \& S. W. Man (Eds.), Research and Endeavors in Moral and Civic Education (pp. 39-58). Hong Kong: Chinese University Press.

Jin, P., Yeung, A. S., Tang, T. O., \& Low, R. (2008). Identifying teachers at risk in Hong Kong: Psychosomatic symptoms and sources of stress. Journal of Psychosomatic Research, 65(4), 357-362.

Kariyawasam, A. G. S. (2002). The Bodhisattva Concept. Kandy: Buddhist Publications Society.

Kennedy, K. J. (2005). Changing schools for changing times: New directions for the school curriculum in Hong Kong. Hong Kong: Chinese University Press.

Kumpfer, K. L. (2002). Factors and processes contributing to resilience. In M. D. Glantz \& J. L., Johnson (Eds.), Resilience and Development (pp. 179-224). US: Springer.

Kwan, T., \& Lopez-Real, F. (2005). Mentors' perceptions of their roles in mentoring student teachers. Asia-Pacific Journal of teacher Education, 33(3), 275-287. http://www.tandfonline.com/doi/pdf/10.1080/13598660500286267

Lam, B. H. (2011). A Reflective Account of a Pre-service Teacher's Effort to Implement Progressive Curriculum in Field Practice. Schools: Studies in Education, 8(1), 22-39.

Lam, B. H. (2011). Teachers as Researchers and teacher Development. In S. N. Phillipson \& B. H. Lam (Eds.), Learning and Teaching in the Chinese Classroom (pp. 265-290). Hong Kong: Hong Kong University Press.

Lam, B. H. (2012). Why do they want to become teachers? A Study on Prospective teachers' Motivation to Teach in Hong Kong. Asian Pacific Education Researcher, 21(2), 307- 314.

Lam, B. H. (2014). Challenges beginning teachers face. Schools: Studies in Education, 11(1), 156169.

Li, J. (2003). The core of Confucian learning. American Psychologist, 58(2), 146-147.

Lopez-Real, F., \& Kwan, T. (2005). Mentors' perceptions of their own professional develop- ment during mentoring. Journal of Education for Teaching, 31(1), 15-24. http://www.tandfonline.com/doi/pdf/10.1080/02607470500043532

McKernan, J., \& McKernan, J. (2013). Curriculum action research: A handbook of methods and resources for the reflective practitioner ( $2 \mathrm{nd}$ ed.). London: Routledge.

McLaughlin, M. W., \& Zarrow, J. (2001). Teachers engaged in evidence-based reform: Trajectories 
of teachers' inquiry, analysis, and action. In A. Lieberman \& L. Miller (Eds.), teachers caught in the action: Professional development that matters, Vol. 31 (pp. 79-101). New York: teachers College Press.

Neary, M. (2000). Supporting students' learning and professional development through the process of continuous assessment and mentorship. Nurse Education Today, 20(6), 463-474.

Nguyen, P. M., Terlouw, C., \& Pilot, A. (2006). Culturally appropriate pedagogy: the case of group learning in a Confucian Heritage Culture context. Intercultural Education, 17(1), 1-19.

Niemiec, C. P., \& Ryan, R. M. (2009). Autonomy, competence, and relatedness in the class- room: Applying self-determination theory to educational practice. Theory and Research in Education, 7(2), 133-144.

Penfold, P., \& van der Veen, R. (2014). Investigating Learning Approaches of Confucian Heritage Culture Students and teachers' Perspectives in Hong Kong. Journal of Teaching in Travel \& Tourism, 14(1), 69-86.

Rhee, E., Uleman, J. S., Lee, H. K., \& Roman, R. J. (1995). Spontaneous self-descriptions and ethnic identities in individualistic and collectivistic cultures. Journal of Personality and Social Psychology, 69(1), 142-152.

Ross, J. A., \& Bruce, C. D. (2007). Teacher self-assessment: A mechanism for facilitating professional growth. Teaching and teacher Education, 23(2), 146-159.

Ryan, R. M., \& Deci, E. L. (2000). Self-determination theory and the facilitation of intrinsic motivation, social development, and well-being. American Psychologist, 55(1), 68-78. http://citeseerx.ist.psu.edu/viewdoc/download?doi=10.1.1.335.6945\&rep=rep1\&type=pdf

Sandholtz, J. H. (2000). Interdisciplinary team teaching as a form of professional development. Teacher Education Quarterly, 27(3), 39-54.

Shulman, L. S. (1986). Those who understand: Knowledge growth in teaching. Educational Research, 15(2), 4-14.

Shulman, L. S. (1987). Knowledge and teaching: foundations of the new reform. Harvard Educational Review, 57(1), 1-22.

Speck, M., \& Knipe, C. (2005). Why can't we get it right? Designing high-quality profession-al development for standards-based schools. Thousand Oaks: Corwin Press.

Stringer, E. T. (2007). Action research (3rd ed.). California: Sage.

Tang, C. S. K., Au, W. T., Schwarzer, R., \& Schmitz, G. (2001). Mental health outcomes of job stress among Chinese teachers: role of stress resource factors and burnout. Journal of Organizational Behavior, 22(8), 887-901.

Thiessen, D. (2000). A skillful start to a teaching career: A matter of developing impactful behaviors, reflective practices, or professional knowledge?. International Journal of Educational Research, 33(5), 515-537.

Triandis, H. C. (2001). Individualism-collectivism and personality. Journal of Personality, 69(6), 907-924.

Twenge, J. M., \& Crocker, J. (2002). Race and self-esteem: Meta-analyses comparing whites, blacks, Hispanics, Asians, and American Indians and comment on Gray-Little and Hafdahl (2000). Psychological Bulletin, 128(3), 371-408.

Villegas-Reimers, E. (2003). Teacher professional development: an international review of the literature. Paris: International Institute for Educational Planning. 
Visiting Panel. (1982). A Perspective on Education in Hong Kong. Report by a Visiting Panel. Retrieved from

Walling, B., \& Lewis, M. (2000). Development of professional identity among professional development school pre-service teachers: longitudinal and comparative analysis. Action in teacher Education, 22(2A), 65-72.

Wise, A. E. (2001). Creating a High-Quality Teaching Force. Educational Leadership, 58(4), 1821.

Zhai, F., Raver, C. C., \& Li-Grining, C. (2011). Classroom-based interventions and teachers' perceived job stressors and confidence: Evidence from a randomized trial in Head Start settings. Early Childhood Research Quarterly, 26(4), 442-452. 
Anexo 1. Evolución de conceptos y actividades de Desarrollo Profesional Docente en Hong Kong

\begin{tabular}{|c|c|c|c|}
\hline & $\begin{array}{l}\text { Comienzos: } \\
\text { Anterior a la década de los } 80 \\
\end{array}$ & $\begin{array}{l}\text { Situación anterior: } \\
\text { Los } 90 \\
\end{array}$ & $\begin{array}{l}\text { Situación actual: } \\
\text { Década del } 2000 \text { en adelante }\end{array}$ \\
\hline \multirow{2}{*}{ 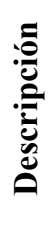 } & PROFESORES COMO TRABAJADORES & $\begin{array}{c}\text { PROFESORES COMO REALIZADORES DE } \\
\text { MULTIPLES TAREAS }\end{array}$ & $\begin{array}{c}\text { PROFESORES COMO APRENDICES } \\
\text { PERMANENTES }\end{array}$ \\
\hline & $\begin{array}{l}\text { El DPD es equiparable en gran medida a 'la } \\
\text { formación inicial y permanente en el conocimiento } \\
\text { disciplinar y pedagógico' }\end{array}$ & $\begin{array}{l}\text { El DPD es considerado como 'entrenamiento en conocimientos } \\
\text { disciplinares, pedagogías, además de las competencias } \\
\text { genéricas realizar múltiples tareas en las escuelas' }\end{array}$ & $\begin{array}{l}\text { El DPD es definido por la ACTEQ como el } \\
\text { aprendizaje permanente autónomo de los } \\
\text { profesores en las comunidades. }\end{array}$ \\
\hline 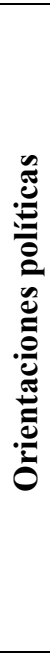 & $\begin{array}{l}\text { - "También deben tenerse en cuenta otros objetivos, } \\
\text { en particular, garantizar un suministro regular de } \\
\text { docentes capacitados para cumplir con plazas } \\
\text { docentes a medida que surgen y se provee de } \\
\text { formación básica a los profesores que ejercen pero } \\
\text { no están capacitados" (Hong Kong Government, } \\
\text { 1978, pág. 13) } \\
\text { "Observamos que las lecciones tendían a ser } \\
\text { centradas en el docente, con el uso de pocos } \\
\text { recursos no más allá de la tiza y la pizarra " } \\
\text { (Visiting Panel, 1982, pág. 51) “... Los estudiantes } \\
\text { pasan muy poco tiempo en pensamiento crítico y } \\
\text { analítico" (pág. 55) "... el sistema escolar debe } \\
\text { centrarse ahora en algunos de los cambios causados } \\
\text { por esta inscripción rápida en alza- las nuevas } \\
\text { cuestiones relacionadas con la calidad más que con } \\
\text { la cantidad" (pág. 56) }\end{array}$ & $\begin{array}{l}\text { " Los colegios (...) deben contribuir al crecimiento personal de } \\
\text { sus estudiantes, ayudándoles a desarrollar un sentido moral y } \\
\text { prepararles para la transición física, emocional y mental de la } \\
\text { edad adulta". (Educational and Manpower Branch, 1993, pág. } \\
\text { 5) } \\
\text { "Las recomendaciones (en este estudio) principalmente se } \\
\text { centran en las formas de mejorar la gestión y el rendimiento } \\
\text { escolar a través de la provisión de la educación escolar de } \\
\text { calidad para conocer mejor las necesidades de los estudiantes. } \\
\text { (...) La EC (Comisión de Educación) encuentra que hay una } \\
\text { necesidad de mejorar la calidad del actual sistema educativo" } \\
\text { (Education Commission, 1997, pág. 5) }\end{array}$ & $\begin{array}{l}\text { - “... La ACTEQ recomienda que los profesores } \\
\text { de instituciones educativas deben ser } \\
\text { constructivos como las comunidades de } \\
\text { aprendizaje favorables para el desarrollo de los } \\
\text { profesores de la capacidad del aprendizaje } \\
\text { permanente. ACTEQ también recomienda un } \\
\text { sistema de prácticas con el fin de proporcionar } \\
\text { nuevos docentes con un entorno completo } \\
\text { propicio para su desarrollo profesional." } \\
\text { (ACTEQ, 2003, p. i) } \\
\text { "La COTAP continuará defendiendo el } \\
\text { desarrollo robusto de nuestra entregada profesión } \\
\text { docente, una profesión de aprendizaje... la labor } \\
\text { docente de calidad y profesional conducirá a la } \\
\text { educación de calidad para la mejora del } \\
\text { aprendizaje de los alumnos. (Committee on } \\
\text { Professional Development of Teachers and } \\
\text { Principals; COTAP, 2014) }\end{array}$ \\
\hline
\end{tabular}




\begin{tabular}{|c|c|c|c|c|}
\hline \multirow{3}{*}{ 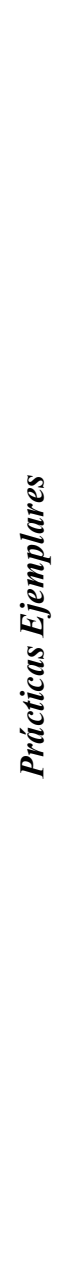 } & 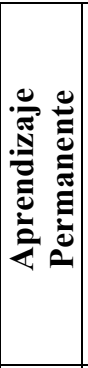 & No aplicable a este período & No aplicable a este período & $\begin{array}{ll}\text { - } & \text { El Marco General de las Competencias de } \\
& \text { Docente (2003) } \\
\text { - } & \text { El Plan de Inducción Docente (2005) } \\
\text { - } & \text { Planes de Lección de Colaboración, Observación } \\
\text { de las lecciones de compañeros, y Programas de } \\
\text { Intercambio. } \\
\text { - Sesiones compartidas dentro y fuera de los } \\
\text { colegios. } \\
\text { - Encuestas sobre la visión de los docentes acerca } \\
\text { de las prácticas de DPD. }\end{array}$ \\
\hline & 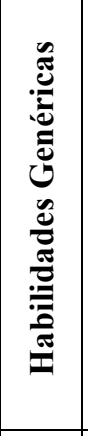 & No aplicable a este período & $\begin{array}{l}\text { - Construir centros de recursos sobre tópicos específicos: } \\
\text { En Centro de Guía para los Profesores en 1995, } \\
\text { relacionado con orientación psicopedagógica para los } \\
\text { estudiantes. } \\
\text { El Centro de Educación de Estudiantes con Altas } \\
\text { Capacidades establecido en 1995 para orientar la } \\
\text { educación de estudiantes superdotados. } \\
\text { Información de Niveles de Competencia Tecnológica para } \\
\text { desarrollar las habilidades tecnológicas de los docentes, } \\
\text { "siendo capaz de usar las TIC como herramientas productivas } \\
\text { para mejorar la eficacia y la efectividad del trabajo del } \\
\text { profesor" (Au et al., 1999) }\end{array}$ & 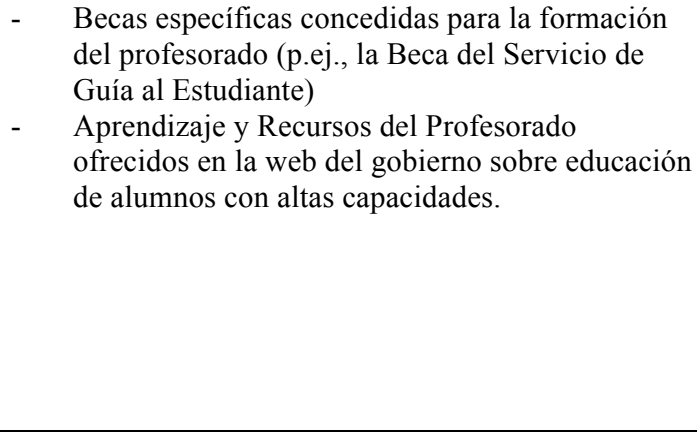 \\
\hline & : & 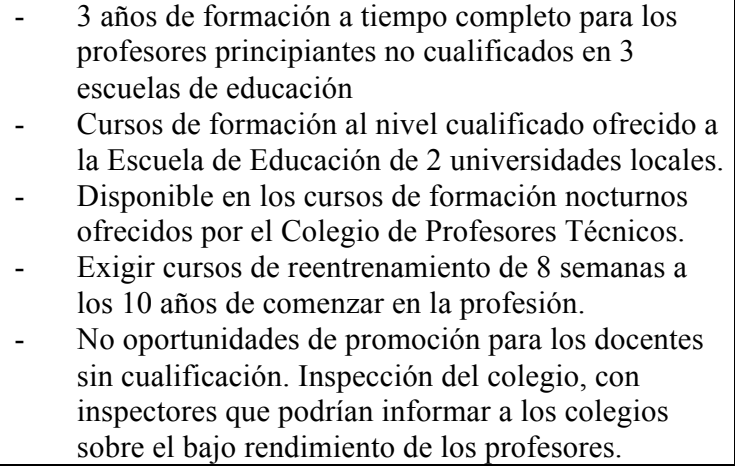 & $\begin{array}{l}\text { - } \quad \begin{array}{l}\text { Continuación de los requisitos previos de formación y } \\
\text { provisión de cursos. }\end{array} \\
\text { - } \\
\text { Requisitos que todos los docentes deben estar al menos } \\
\text { graduados (1997). } \\
\text { Número de plazas en formación docente en el grado o por } \\
\text { encima del nivel aumentado (1998). } \\
\text { - Cursos de asesoramiento y orientación a tiempo completo o } \\
\text { parcial que se ofrecen a los docentes de las principales } \\
\text { universidades. } \\
\text { Cursos ofrecidos para los requisitos de competencias } \\
\text { específicas y el examen de la competencia (p.ej., informática, y } \\
\text { dominio del idioma). }\end{array}$ & $\begin{array}{l}\text { - } \quad \text { Continuación de la formación previa ofrecida por } \\
\text { las universidades principales. } \\
\text { - Talleres de tutoría, seminarios y cursos provistos } \\
\text { para tutores docentes. } \\
\text { - Cursos, talleres y seminarios provistos para } \\
\text { docentes sobre el plan de lección de colaboración } \\
\text { y la lección de observación. } \\
\text { - Cursos de formación sobre la coordinación del } \\
\text { DPD para la gestión de la escuela. } \\
\text { - Docentes motivados para obtener el postgrado } \\
\text { (Máster y doctorado) en educación. } \\
\text { - La Formación en Liderazgo TI (desde } 2000 \text { en } \\
\text { adelante). }\end{array}$ \\
\hline
\end{tabular}

